\title{
Sediments of soft spheres arranged by effective density
}

Nature Materials 10, 716 (2011)

Authors: César Gonzáles Serrano, Joseph J. McDermott, and Darrell Velegol

Recommended and a commentary by Marjolein Dijkstra, Utrecht University

In this paper, César Gonzáles Serrano, Joseph J. McDermott, and Darrell Velegol showed in a beautiful and carefully conducted series of experiments that binary and five-component mixtures of charged colloids separate spontaneously into distinct layers in sedimentation. The spontaneous formation of separated layers can serve as a useful tool to separate different colloidal components.

Gravity is often non-negligible in colloidal suspensions, as the gravitational energy becomes comparable to the thermal energy for colloid sizes of about a micrometer. Hence, a spatial inhomogeneous suspension characterised by a density profile $\rho(z)$ that varies with altitude $z$ is obtained due to the gravitational field, which is frequently considered to be a nuisance in experiments.

However, one can also take advantage of gravity. In the case of a very dilute colloid concentration or at sufficiently high altitudes, the system behaves like an ideal gas and the system obeys the theoretically predicted barometric law $\rho(z) \propto \exp \left(-m g z / k_{B} T\right)$ with $k_{B}$ Boltzmann's constant, $T$ the temperature, $m$ the buoyancy mass of the particles, and $g$ the gravitational acceleration. This Boltzmann distribution follows from a competition between minimal energy (all colloids at the bottom) and maximum entropy (a homogeneous distribution in the available volume). In 1910, Jean Perrin measured such a density profile under the microscope which enabled him to determine Boltzmann's constant and hence, Avogadro's number [1]. Exploiting the analogy between colloids and atoms, Perrin convinced for once and for all, even the most prominent sceptics, the existence of atoms and molecules. He received the Nobel Prize in 1926. The barometric distribution is only valid for dilute dispersions, in which colloid interactions can be ignored.

More recently, sedimentation has received renewed attention, as it was realized that the hard-sphere bulk equation of state can be measured in a large density range from a single measurement of the sedimentation profile of a dense suspension of colloidal hard spheres $[2,3]$.

Quite some attention has also been devoted to sedimentation equilibria of charged colloids in an electrolyte solution with cations and anions, in particular under conditions of low salinity, where strong deviations from the barometric distribution have been found [4-6]. The most striking phenomenon is that the distribution of colloids with a charge $Z e$ extends to much higher altitudes than is to be expected on the basis of their buoyant mass. The mean height of charged colloids is increased by a factor $Z$, i.e., from $L$ in the barometric (high salt) regime to $Z L$ in the deionized case with $L=k_{B} T / m g$ the gravitational length. The force that lifts the colloids against gravity is provided by an electric field that is induced by spontaneous charge separation over macroscopic distances. Although this phenomenon costs energy, the system gains entropy because of a more homogeneous distribution of salt ions (and colloids). 
The same lift mechanism persists also in the case of binary and multicomponent mixtures of charged colloids at low salinity. More surprisingly, the lifting effect can also result in a segregation effect in these mixtures, whereby layers of essentially pure species $i$ are formed at an average height given by the charge per mass $Z_{i} L_{i}[7,8]$. This effect appears due to a competition between ionic entropy, gravitational energy, and electrostatic energy, and can be understood from a perspective, where the colloids and ions are treated on the same footing as well as from a perspective, which is based on a much simpler colloids-only perspective using effective (screened-Coulomb) interactions [8].

This intriguing lifting and segregation effect has been observed now by César Gonzáles Serrano, Joseph J. McDermott, and Darrell Velegol, in a series of experiments on binary and five-component systems of micron-sized sulphate-functionalized polystyrene latex (PSL) particles and silica particles. They observe that the suspensions separate spontaneously into distinct layers on sedimentation, which can serve as a route to sort particles by charge per mass. To exploit this segregation effect to sort colloidal particles, a better understanding is required for which parameters (height of the sample, packing fractions of species $i$, charge per mass $Z_{i} L_{i}$ ) the segregation results in nearly pure layers of species $i$.

[1] J. Perrin, J. Phys. 9, 5 (1910).

[2] R. Piazza, T. Bellini, and V. Degiorgio, Phys. Rev. Lett. 71, 4267 (1993).

[3] M. A. Rutgers et al., Phys. Rev. B 53, 5043 (1996).

[4] T. Biben and J.P. Hansen, J. Phys. Condens. Matter 6, A345 (1994).

[5] R. van Roij, , J. Phys. Condens. Matter 15, S3569 (2003).

[6] M. Rasa and A. Philipse, Nature 429, 857 (2004).

[7] J. Zwanikken and R. van Roij, Europhys. Lett. 71, 480 (2005).

[8] A.Cuetos et al., Physical Review E 73, 061402 (2006). 